\title{
Corneal Pachymetry measured with Pentacam and CorvisST in Normal and Keratoconic Eyes
}

\author{
José M González-Méijome, Daniela Lopes-Ferreira, Laura Rico-del-Viejo, Patrícia Neves \\ Helena Ferreira, José Salgado-Borges
}

\begin{abstract}
Purpose: To evaluate the agreement of pachymetry data provided by a new instrument to measure intraocular pressure (IOP) and the corneal biomechanical response with the values provided by Pentacam in healthy and keratoconic eyes.
\end{abstract}

Methods: Fifty-four right eyes from 54 healthy patients (32 females, 22 males) and 82 eyes from 43 keratoconus patients (20 females, 23 males) were included in the study. All patients were evaluated with Pentacam and CorvisST (Oculus, Wetzlar, Germany) in random order in the same session. CorvisCCT was compared to Pentacam pachymetry in at the corneal center (PentacamCCT) and at the thinnest point (PentacamMinCT).

Results: Average pachymetry provided by CorvisST was $534 \pm 34$ microns in the healthy population and $476 \pm 56$ microns in the population with keratoconus. Pachymetric values obtained with Pentacam were highly correlated between them as well as with the Corvis value in both groups, but the correlation coefficients were higher in the healthy group.

Conclusion: Corvis pachymetry can be used interchangeably with Pentacam thinnest point pachymetry in healthy corneas. However, in keratoconic corneas, the difference between both parameters will be higher as the disease progresses and increases the difference between Pentacam pachymetry at cornea center and at the thinnest point.

Keywords: Pachymetry, Scheimpflug photography, Keratoconus.

How to cite this article: González-Méijome JM, Lopes-Ferreira D, Rico-del-Viejo L, Neves P, Ferreira H, Salgado-Borges J. Corneal Pachymetry measured with Pentacam and CorvisST in Normal and Keratoconic Eyes. Int J Kerat Ect Cor Dis 2013;2(3):104-107.

Source of support: The authors want to thankAntonio Moutinho Lda and Oculus for the loan of the corvis ST. The authors have no proprietary interest in any of the devices mentioned in this article. Data presented in part at ESCRS Meeting, Amsterdam 5-9 October 2013.

\section{Conflict of interest: None}

\section{INTRODUCTION}

Central corneal thickness (CCT) parameter is paramount in several clinical and research applications. ${ }^{1}$ Accurate measurements of corneal thickness are crucial in several surgical applications ${ }^{2-4}$ and in the interpretation of the intraocular pressure (IOP) values measured with most tonometric techniques given the relationship between CCT and corneal resistance to applannation. ${ }^{1,5}$ Several contact and noncontact instruments provide a measurement of the CCT, some are solely intended to obtain this parameter, ${ }^{6}$ most of them integrated in instruments aiming a more complete description of the cornea ${ }^{7-10}$ or to obtain other ocular parameters. $^{11-13}$

A new instrument is available to clinically measure corneal biomechanical properties, the CorvisST (Oculus, Wetzlar, Germany). This instrument obtains a high-speed sequence of scheimpflug images of the anterior segment of the eye while the cornea is bended under pneumatic force to obtain a value of intraocular pressure (IOP) and a single value of CCT. ${ }^{14}$ This value might be clinically relevant to correct the IOP values provided by CorvisST. However, although this CCT value is based on the same principle as other validated methods, such as Pentacam and Sirius, ${ }^{10 ; 15}$ to the best of our knowledge no previous study has addressed its agreement with one of those systems. We hypothesize that while the agreement between the two instruments might be good for healthy corneas, it might be less precise in diseased corneas where the thinnest point is significantly deviated from the geometric center.

Thus, the purpose of this study was to evaluate the agreement of the CCT value provided by CorvisST with Pentacam readings in normal and keratonic corneas.

\section{METHODS}

Fifty-four healthy volunteers (32 females and 22 males; age: $73 \pm 6$ years) and forty-three kerartoconus patients (20 females and 23 males; age: $37 \pm 12$ years) were recruited to participate in this study. Inclusion criteria required that the subjects were free of ocular disease except keratoconus, were not wearing contact lenses for at least 12 hours prior to the measurements and were not under systemic or topical medication. The study protocol was reviewed and approved by the CHEDV Institutional Review Board. In agreement with the Declaration of Helsinki volunteers signed a consent form after the purpose and methods of the study were explained to them. In the healthy group, only the right eye of each patient was measured, in the keratoconus patients, both eyes were measured except when one of the eyes had been subjected to a surgical procedure such as corneal cross-linking, intracorneal ring segment implantation or keratoplasty.

Pentacam and CorvisST measures were done in random order by two different trained technicians. Three repeated measures of CorvisST (CorvisCCT) were conducted in each patient and averaged. For Pentacam, three values of 

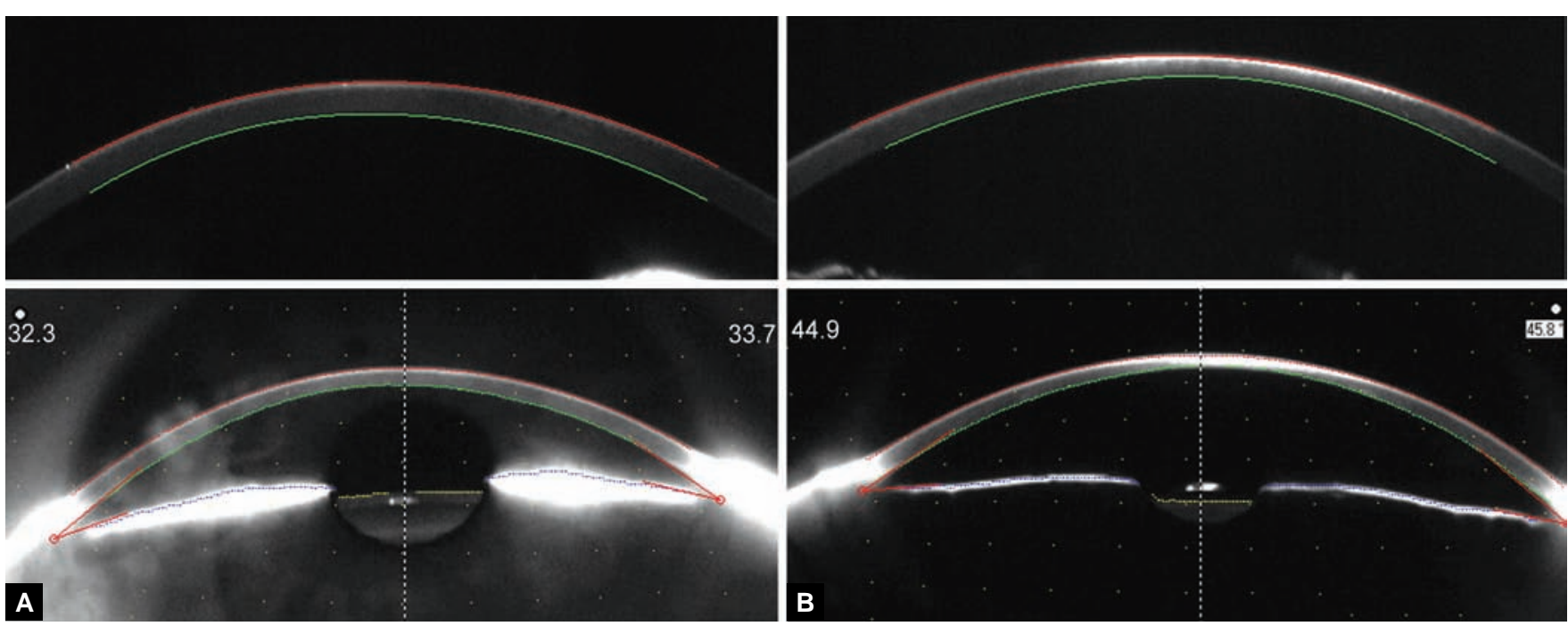

Figs 1A and B: Scheimpflug images from CorvisST over $8 \mathrm{~mm}$ diameter (top) and Pentacam over $12 \mathrm{~mm}$ (bottom) in a thin healthy cornea $(A)$ and a keratoconic cornea with some degree of scarring (B)

Table 1: Mean values, comparisons and correlations between Pentacam and Corvis pachymetric in the two clinical groups (healthy and keratoconic corneas) and comparisons among them

\begin{tabular}{|c|c|c|c|c|}
\hline \\
\hline & & $\begin{array}{l}\text { Healthy } \\
(n=54)\end{array}$ & $\begin{array}{c}\text { Keratoconus } \\
\quad(n=82)\end{array}$ & $\begin{array}{c}\text { Significance } \\
\text { between groups } \\
\left(\text { Sig. }^{+}\right)\end{array}$ \\
\hline \multirow[t]{3}{*}{ Mean \pm SD } & CorvisCCT & $534 \pm 34$ & $475 \pm 56$ & $p=0.002$ \\
\hline & PentacamCCT & $539 \pm 35$ & $486 \pm 56$ & $p=0.006$ \\
\hline & PentacamMinCT & $536 \pm 35$ & $458 \pm 62$ & $p=0.001$ \\
\hline \multirow[t]{3}{*}{ Differences within groups (Sig. $\ddagger$ ) } & PentacamCCT-PentacamMinCT & $\begin{array}{c}3.0 \pm 2.1 \\
(p<0.001)\end{array}$ & $\begin{array}{l}27.7 \pm 24.9 \\
(p<0.001)\end{array}$ & $p<0.001$ \\
\hline & PentacamCCT-CorvisCCT & $\begin{array}{l}4.3 \pm 10.9 \\
(p=0.005)\end{array}$ & $\begin{array}{l}11.0 \pm 20.9 \\
(p<0.001)\end{array}$ & $p=0.034$ \\
\hline & PentacamMinCT - CorvisCCT & $\begin{array}{l}1.4 \pm 11.7 \\
(p>0.05)\end{array}$ & $\begin{array}{c}-16.7 \pm 21.8 \\
(p<0.001)\end{array}$ & $p=0.001$ \\
\hline \multirow[t]{3}{*}{ Correlations } & PentacamCCT vs MinCT & $\begin{array}{c}0.998 \\
(p<0.001)\end{array}$ & $\begin{array}{c}0.916 \\
(p<0.001)\end{array}$ & - \\
\hline & PentacamCCT vs CorvisCCT & $\begin{array}{c}0.950 \\
(p<0.001)\end{array}$ & $\begin{array}{c}0.931 \\
(p<0.001)\end{array}$ & - \\
\hline & PentacamMinCT vs CorvisCCT & $\begin{array}{c}0.942 \\
(p<0.001)\end{array}$ & $\begin{array}{c}0.938 \\
(p<0.001)\end{array}$ & - \\
\hline
\end{tabular}

ССT: central corneal thickness; MinCT: minimum corneal thickness; ${ }^{\dagger}$ statistical significance for differences between healthy and keratoconic groups (independent sample T-test); ${ }^{\ddagger}$ statistical significance for differences between measures within the same group (paired sample T-test)

the central corneal thickness (PentacamCCT) and minimum corneal thickness (PentacamMinCT) were recorded. A total of 54 healthy eyes and 82 keratoconus eyes were included in the final sample for subsequent analysis. Figures $1 \mathrm{~A}$ and B shows the Scheimpflug images from CorvisST and Pentacam in a normal and a keratoconic eye.

Statistical analysis was conducted using SPSS v.20.0. Normality of data distribution was assessed using Kolmogorov-Smirnov test. Comparison between both instruments within each group was conducted by repeated samples T-test, while correlation between measures was done by Pearson's correlation. Comparisons between groups were performed with independent samples T-test to compare the behavior of keratoconus and healthy corneas regarding CCT measures. Agreement between techniques within each group was assessed by Bland-Altman analysis. The level of statistical significance was set at $\alpha \leq 0.05$.

\section{RESULTS}

Table 1 presents the comparison between healthy and keratoconus groups for the average of pachymetric data as well as the differences between the 3 parameters recorded. As expected, pachymetric values were significantly different between healthy and keratoconic corneas. Difference between CCT and MinCT obtained with Pentacam was significantly higher in the keratoconus group. Pachymetric values obtained with Pentacam were highly correlated between them as well as with the Corvis value in both groups, but the correlation coefficients were higher in the healthy group. Differences between CorvisCCT and PentacamCCT or PentacamMinCT were also significantly higher in the keratoconus group.

Bland-Altman analysis for the differences between Pentacam and CorvisST is presented in Figures 2 A to D. Differences between CorvisCCT and PentacamCCT were 
statistically significant $(p=0.005)$ while differences between CorvisCCT and PentacamMinCT were not $(p=0.399)$ in the healthy group. In the keratoconus group differences between CorvisCCT and PentacamCCT or PentacamMinCT were statistically significant $(\mathrm{p}<0.001)$.

Differences between Pentacam and Corvis pachymetry were correlated with the differences between PentacamCCT and PentacamMinCT. This evaluation attempted to evaluate if the agreement between both instruments as a function of the deviation between CCT and MinCT. According to this analysis, the higher the asymmetry in the PentacamCT (PentacamCCT-PentacamMinCT), the higher the deviation of Corvis pachymetry from PentacamCCT in the healthy and keratoconic group $(r=0.292 ; p=0.032$ and $r=0.562$; $p<0.001$, respectively) and Corvis pachymetry from PentacamMinCT $(\mathrm{r}=0.453 ; \mathrm{p}=0.001$ and $\mathrm{r}=0.608 ; \mathrm{p}<$ 0.001 respectively).

\section{DISCUSSION}

The present work aimed to evaluate the agreement between Corvis pachymetry and pachymetry at center and the thinnest point provided by Pentacam. Conversely, the same
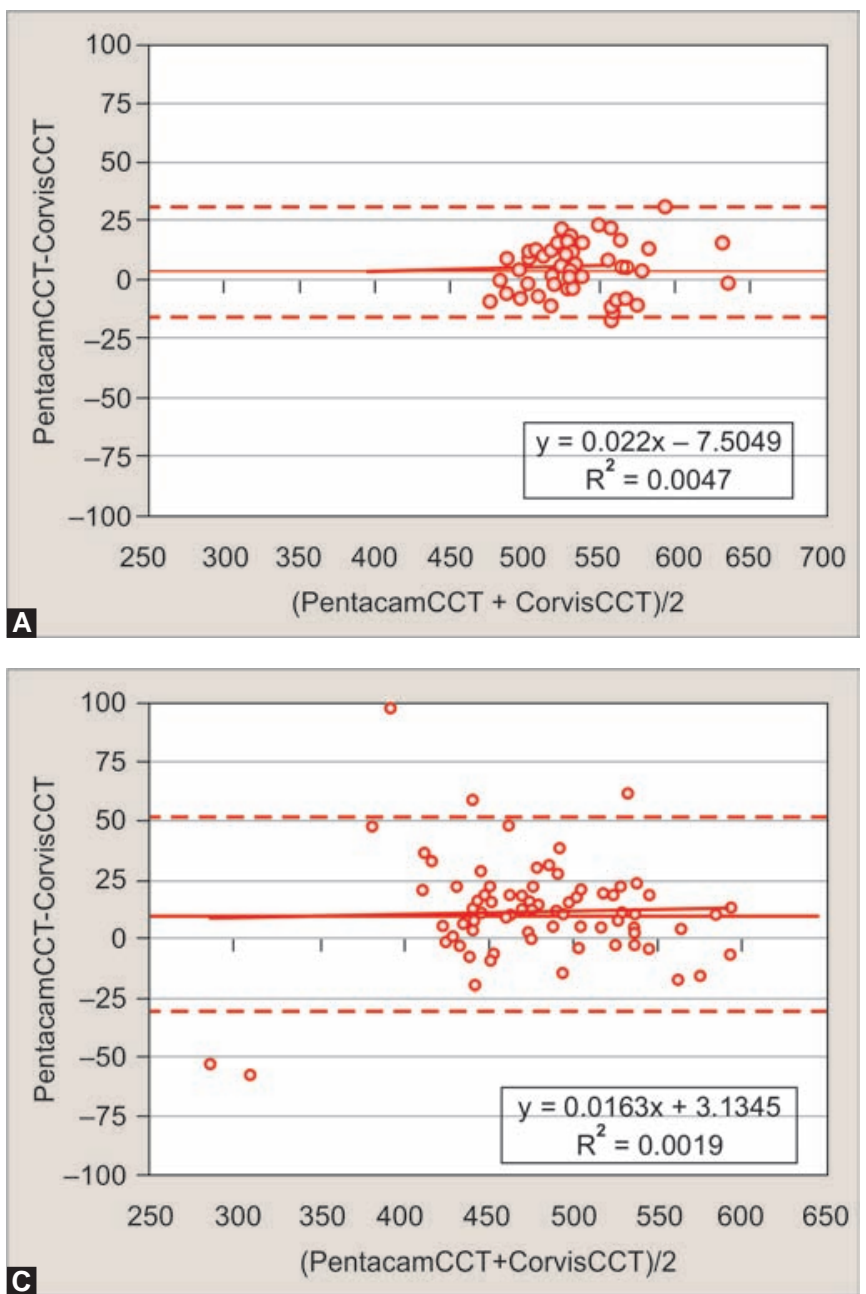

agreement could not be observed in keratoconic eyes. Considering that the evaluation of biomechanical properties of the cornea is more relevant in diseased corneas, the results of the present study suggest that the use of pachymetric values provided by Corvis should be considered carefully in these cases, particularly when there is a large difference between PentacamCCT and Pentacam MinCT which should be the case in moderate and advanced keratoconus. ${ }^{16}$

The immediate consequence of the present results is the implication in the correction of tonometric values using corneal thickness. Considering that for every 20 microns of deviation from the average corneal thickness (545 microns) should be compensated by about $1 \mathrm{~mm} \mathrm{Hg}$ in the tonometric result, the highest difference in the healthy population should be of this order of magnitude as the $95 \%$ confidence interval for differences between Corvis and PentacamCCT values is within the range of \pm 25 microns. In the keratoconic population, differences can go up to 3 times that of the normal population. So, an approximate estimation might be derived from the Corvis pachymetry but an accurate correction will require Pentacam pachymetry. In the keratoconic group, while most of the differences should
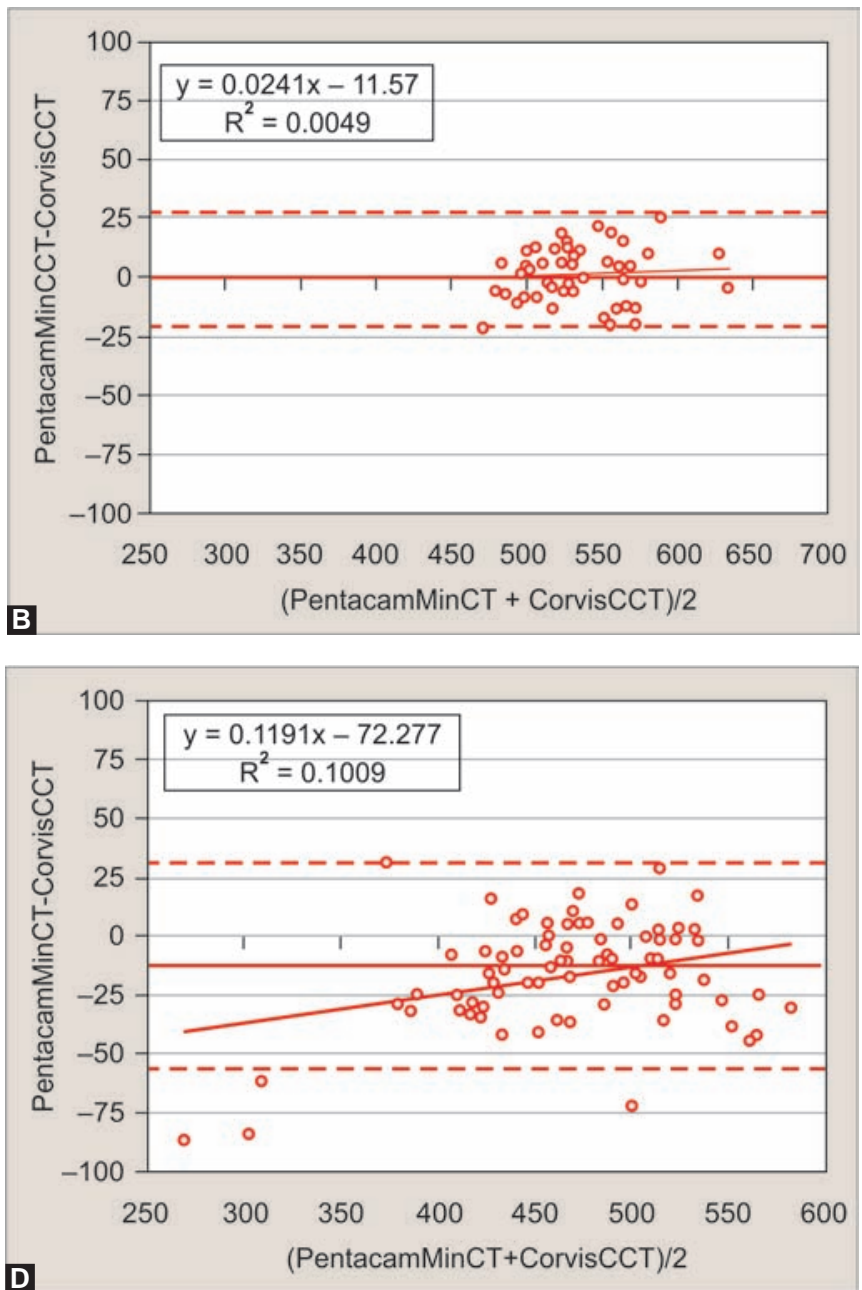

Figs 2 A to D: Bland-Altman analysis for differences of CorvisCCT with PentacamCCT and PentacamMinCT in healthy corneas (A and B) and keratoconic corneas ( $C$ and $D)$ 
remain within a narrow range when comparing CorvisCCT and PentacamCCT, about $25 \%$ of the subjects would depart from each other by over 20 microns as shown in Figure 2C. In these cases, it is expected that CorvisST would significantly underestimate corneal thickness compared to the PentacamCCT values. As expected PentacamMinCT is comparable to PentacamCCT in normal eyes and as expected is systematically lower in keratoconic corneas.

Despite the differences obtained between CorvisST and Pentacam regarding the measurements of the CCT, CorvisCCT can be used as a screening value either for keratroconus or normal subjects. Other values provided by CorvisST, should not be affected by these differences as they reflect geometrical measures of radius, distances and speed to characterize the biomechanical response of the cornea to an air puff. ${ }^{14,17}$ For the purpose of intraocular pressure (IOP) correction, the value provided by CorvisST should allow to estimate actual values of IOP within a range of $\pm 1.00 \mathrm{~mm} \mathrm{Hg}$ from the values corrected by PentacamCCT. Conversely, in about $25 \%$ of the keratoconic corneas, this correction should exceed the $1.00 \mathrm{~mm} \mathrm{Hg}$ difference, being much higher in $10 \%$ of the eyes who are those showing differences between PentacamCCT and CorvisCCT higher than \pm 50 microns.

\section{REFERENCES}

1. Doughty MJ, Zaman ML. Human corneal thickness and its impact on intraocular pressure measures: a review and metaanalysis approach. Surv Ophthalmol 2000;44:367-408.

2. Greenstein SA, Shah VP, Fry KL, Hersh PS. Corneal thickness changes after corneal collagen crosslinking for keratoconus and corneal ectasia: one-year results. J Cataract Refract Surg 2011;37:691-700.

3. Ho T, Cheng AC, Rao SK, et al. Central corneal thickness measurements using Orbscan II, Visante, ultrasound, and Pentacam pachymetry after laser in situ keratomileusis for myopia. J Cataract Refract Surg 2007;33:1177-1182.

4. Kim SW, Byun YJ, Kim EK, Kim TI. Central corneal thickness measurements in unoperated eyes and eyes after PRK for myopia using Pentacam, Orbscan II, and ultrasonic pachymetry. J Refract Surg 2007;23:888-894.

5. Patel S, McLaughlin JM. Effects of central corneal thickness on measurement of intraocular pressure in keratoconus and postkeratoplasty. Ophthalmic Physiol Opt 1999;19:236-241.

6. Queiros A, Gonzalez-Meijome JM, Fernandes P, et al. Technical note: accuracy and repeatability of a new portable ultrasound pachymeter. Ophthalmic Physiol Opt 2007;27:190-193.

7. Gonzalez-Perez J, Gonzalez-Meijome JM, Rodriguez Ares MT, Parafita MA. Topographic paracentral corneal thickness with pentacam and orbscan: effect of acoustic factor. Eye Contact Lens 2011;37:348-353.

8. Rosa N, Lanza M, Borrelli M, et al. Comparison of central corneal thickness measured with Orbscan and Pentacam. J Refract Surg 2007;23:895-899.

9. Gonzalez-Meijome JM, Cervino A, Yebra-Pimentel E, Parafita MA. Central and peripheral corneal thickness measurement with
Orbscan II and topographical ultrasound pachymetry. J Cataract Refract Surg 2003;29:125-132.

10. Jorge J, Rosado J, Diaz-Rey J, Gonzalez-Meijome J. Central corneal thickness and anterior chamber depth measurement by Sirius((R)) Scheimpflug tomography and ultrasound. Clin Ophthalmol 2013;7:417-422.

11. Yazici AT, Bozkurt E, Alagoz C, et al. Central corneal thickness, anterior chamber depth, and pupil diameter measurements using Visante OCT, Orbscan and Pentacam. J Refract Surg 2010;26:127-133.

12. Lam AK, Chen D. Pentacam pachometry: comparison with noncontact specular microscopy on the central cornea and intersession repeatability on the peripheral cornea. Clin Exp Optom 2007;90:108-114.

13. Gonzalez-Perez J, Gonzalez-Meijome JM, Rodriguez Ares MT, Parafita MA. Central corneal thickness measured with three optical devices and ultrasound pachometry. Eye Contact Lens 2011;37:66-70.

14. Hon Y, Lam AK. Corneal deformation measurement using Scheimpflug noncontact tonometry. Optom Vis Sci 2013;90:e1-e8.

15. McAlinden C, Khadka J, Pesudovs K. A comprehensive evaluation of the precision (Repeatability and Reproducibility) of the Oculus Pentacam HR. Invest Ophthalmol Vis Sci 2011;52:7731-7737.

16. Romero-Jimenez M, Santodomingo-Rubido J, GonzalezMeijome JM. The thinnest, steepest and maximum elevation corneal locations in noncontact and contact lens wearers in keratoconus. Cornea 2013;32:332-337.

17. Nemeth G, Hassan Z, Csutak A, et al. Repeatability of ocular biomechanical data measurements with a scheimpflugbased noncontact device on normal corneas. J Refract Surg 2013;29:558-563.

\section{ABOUT THE AUTHORS}

\section{José M González-Méijome (Corresponding Author)}

Associate Professor, CEORLab, Department of Physics and Center of Physics, University of Minho, Braga, Portugal, Phone: +351253604072 e-mail: jgmeijome@fisica.uminho.pt

\section{Daniela Lopes-Ferreira}

CEORLab, Department of Physics and Center of Physics, University of Minho, Braga, Portugal

\section{Laura Rico-del-Viejo}

CEORLab, Department of Physics and Center of Physics, University of Minho, Braga, Portugal

\section{Patrícia Neves}

Department of Ophthalmology, CHEDV, St. M Feira, Portugal

\section{Helena Ferreira}

Department of Ophthalmology, CHEDV, St. M Feira, Portugal

\section{José Salgado-Borges}

Professor, Department of Ophthalmology, CHEDV, St. M Feira Portugal and Hospital Escola Universidade Fernando Pessoa Gondomar, Portugal 